\title{
Eyelid Fissure Narrowing after Recession of the Medial Rectus Muscle
}

\author{
Hirohiko Kakizaki ${ }^{{ }^{*}}$, Yasuhiro Takahashi ${ }^{1}$, Akihiro Ichinose ${ }^{2}$, Masayoshi Iwaki ${ }^{1}$ \\ ${ }^{1}$ Department of Ophthalmology, Aichi Medical University, Nagakute, Aichi, Japan \\ ${ }^{2}$ Department of Plastic Surgery, Kobe University, Chuo, Kobe, Japan \\ E-mail: ${ }^{*}$ cosme@d1.dion.ne.jp \\ Received January 13, 2011; revised February 5, 2011; accepted March 12, 2011
}

\begin{abstract}
A 67-year-old male had suffered from eye movement disturbance from Graves' orbitopathy. His right eye was fixed in an esotropic position. Examination by synoptophore showed that his right eye deviation was 22 degrees medially. The upper eyelid margin reflex distance (MRD) was $3.5 \mathrm{~mm}$ OD and $5.6 \mathrm{~mm}$ OS, and the lower eyelid MRD was $5.1 \mathrm{~mm}$ OU. Six mm medial rectus muscle recession with tenotomy was performed in the patient. The next day after the surgery, his eye position did not change and the right eyelid fissure demonstrated narrowing with upper eyelid lowering and lower eyelid elevation. The right upper eyelid MRD was decreased to $1.9 \mathrm{~mm}$ and the right lower eyelid MRD was similarly decreased to $4.3 \mathrm{~mm}$. On the same day, $4 \mathrm{~mm}$ resection of the ipsilateral lateral rectus muscle was performed, but the same MRDs were observed postoperatively. Patients undergoing medial rectus muscle recession in Graves' orbitopathy need to be informed of this potential complication.
\end{abstract}

Keywords: Graves’ Orbitopathy, Lower Eyelid Elevation, Margin Reflex Distance, Medial Rectus Muscle, Upper Eyelid Lowering

\section{Introduction}

Surgery on the medial rectus muscle is known to have the complication of a change in eyelid fissure height [1]. Recession tends to show widening of the eyelid fissure height, and then narrowing which is mostly caused by resection [1]. Although these results are mainly due to changes in the lower eyelid position, upper eyelid lowering by recession is a rare response [1].

We report a case with eyelid fissure height narrowing with upper eyelid lowering and lower eyelid elevation after medial rectus muscle recession in Graves' orbitopathy.

\section{Case Report}

A 67-year-old male had suffered from eye movement disturbance from Graves' orbitopathy. At first admission, his thyroid hormone level was normal and his clinical activity score [2] was 0 points. He was administered two times steroid pulse therapy more than 1 year prior to the admission. His visual acuity was 0.6 OD and 0.9 OS, the loss of which was caused by a cataract. Hertel exoph- thalmometry showed $15 \mathrm{~mm}$ OU. His right eye was fixed in an esotropic position with a Hirschberg angle of more than 15 degrees (Figure 1(a)). A binocular single vision field [3] showed no single vision area at the time, but he suppressed his right eye under the unconscious. A forced duction test demonstrated lateral restriction but it did not show any medial resistance. According to a synoptophore, his right eye deviation was 22 degrees medially. The upper eyelid margin reflex distance (MRD) was 3.5 $\mathrm{mm}$ OD and $5.6 \mathrm{~mm}$ OS, and the lower eyelid MRD was $5.1 \mathrm{~mm}$ OU (Figure 1(a)).

Six mm medial rectus muscle recession with tenotomy was initially performed in the patient. The next day after surgery, his eye position did not change, but the right eyelid fissure height demonstrated narrowing with upper eyelid lowering and lower eyelid elevation. The right upper eyelid MRD was decreased to $1.9 \mathrm{~mm}$, but the left upper eyelid MRD remained at $5.6 \mathrm{~mm}$. In addition, the right lower eyelid MRD was decreased to $4.3 \mathrm{~mm}$ but the left lower eyelid MRD remained at $5.1 \mathrm{~mm}$. On the same day, $4 \mathrm{~mm}$ advancement of the ipsilateral lateral rectus muscle was performed, but unfortunately, the same MRDs were observed postoperatively (Figure 1(b)). Six 
months later, the right upper and lower eyelid MRDs had slightly improved (upper: $2.3 \mathrm{~mm}$, lower $4.7 \mathrm{~mm}$ ). The left upper eyelid MRD was still $5.6 \mathrm{~mm}$ and that of the lower eyelid was $5.1 \mathrm{~mm}$, which had consistently been at the same levels (Figure 1(c)).

\section{Discussion}

We report a rare case with eyelid fissure narrowing after medial rectus muscle recession in Graves' orbitopathy [1]. Although medial rectus muscle recession tends to show widening of the eyelid fissure height, mostly with lower eyelid lowering [1], our patient showed a paradoxical response by the same procedure.

The medial rectus muscle and the upper and lower eyelids have a close relationship via the medial rectus capsulopalpebral fascia (mrCPF) [4]. The mrCPF contains the medial rectus muscle pulley, the medial check ligament and fibers to the lacrimal caruncle, and it reaches the medial aspect of the upper and lower tarsal plates [4]. The mrCPF enables synchronous movement of the eyeball, eyelids and caruncle [5]. Various tensions of the mrCPF may be reflected in the upper and lower eyelid positions. This is supported by the fact that horizontal eye muscle surgeries can change the position of the eyelids [1].

The medial rectus muscle of our patient was very hardened by fibrosis as shown by the restricted forced duction test. However, the lateral rectus muscle did not demonstrate any restriction during the forced duction test. This finding may be the reason why lateral rectus muscle advancement may not have influenced the postoperative eyelid height.

The clothes lining effect is occasionally demonstrated in the lower eyelids [6]. This is similar to lower eyelid retraction, typically occurring with too much horizontal tension during horizontal eyelid tightening surgery. In this situation, the lower eyelid is not elevated superiorly, but it is more lowered. This phenomenon tends to occur in a more proptotic eye. However, since our patient showed $15 \mathrm{~mm}$ exophthalmos, which is a normal level, lower eyelid elevation may have occurred instead of the clothes lining phenomenon. The same mechanism may be applied to the upper eyelid lowering in this patient.

A previous report [1] that investigated eyelid fissure height change after horizontal rectus muscle surgeries excluded orbital diseases, and it probably also excluded Graves’ orbitopathy. In general, orbital tissues in Graves’ orbitopathy tend to be hardened by fibrosis [7]. This may be the reason why our patient showed a paradoxical outcome compared with the previous report [1].

Our patient showed a large angle esotropia (22 degrees), which was caused by fibrosis of the right medial

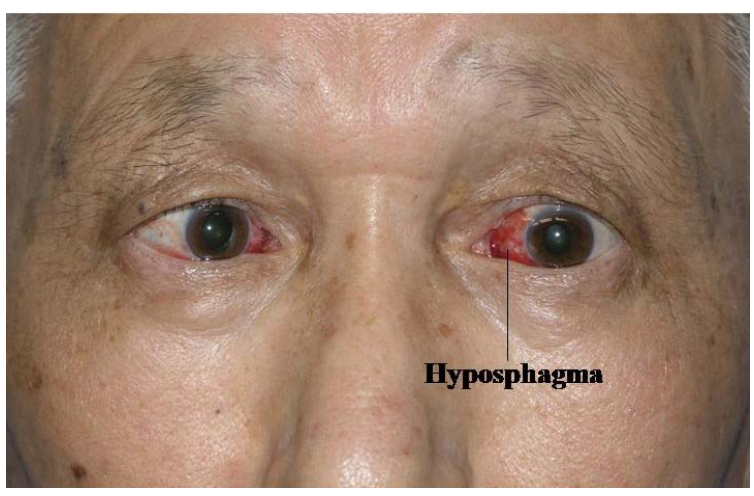

(a)

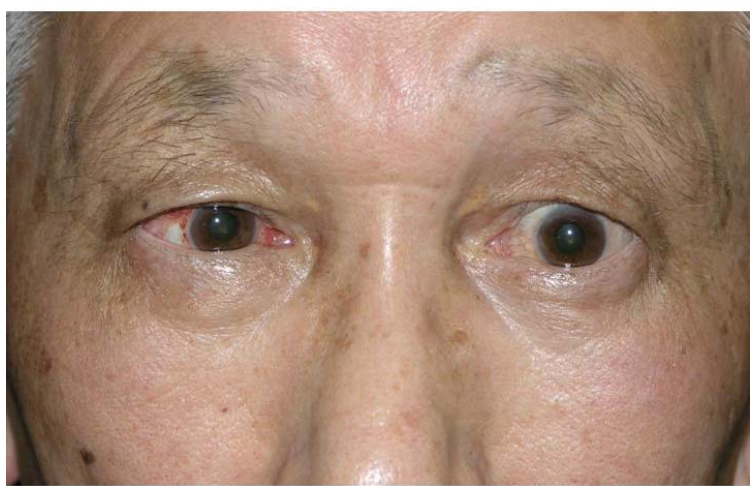

(b)

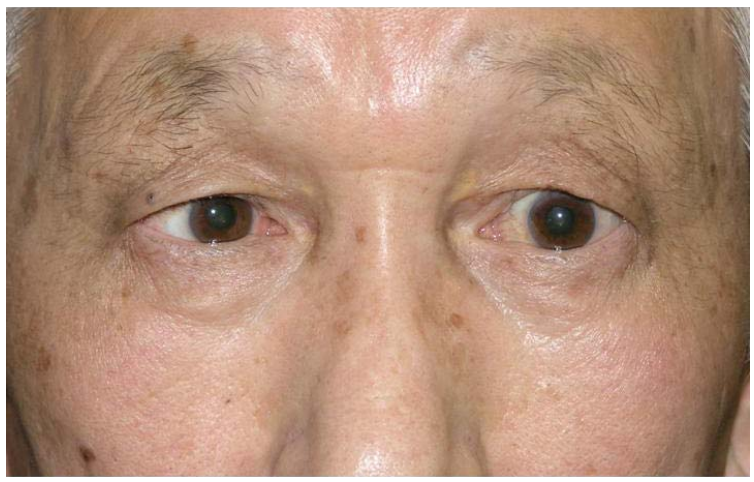

(c)

Figure 1. (a) Preoperative photograph of the patient. The right eye is fixed in an esotropic position with a Hirschberg angle of more than 15 degrees. The upper eyelid margin reflex distance (MRD) is $3.5 \mathrm{~mm} \mathrm{OD}$ and $5.6 \mathrm{~mm} \mathrm{OS}$, and the lower eyelid MRD is $5.1 \mathrm{~mm}$ OU. Subconjunctival hemorrhage of the left eye is not related to any traumas or surgeries; (b) Postoperative 1-month photograph of the same patient. The right eyelid fissure height is narrowed with upper eyelid lowering and lower eyelid elevation. The right upper eyelid MRD is decreased to $1.9 \mathrm{~mm}$ but the left upper eyelid MRD remains at $5.6 \mathrm{~mm}$. The right lower eyelid MRD is decreased to $4.3 \mathrm{~mm}$ but the left lower eyelid MRD remains at $5.1 \mathrm{~mm}$; (c) Postoperative 6-month photograph of the same patient. The right upper and lower eyelid MRDs have slightly improved (upper: $2.3 \mathrm{~mm}$, lower $4.7 \mathrm{~mm}$ ). The left upper eyelid MRD is still $5.6 \mathrm{~mm}$ and that of the lower eyelid is $5.1 \mathrm{~mm}$. 
rectus muscle. Although he ideally should have been operated on with a bilateral medial rectus muscle recession, he hoped to only have an operation for the affected eye. Therefore, we only operated on his right eye. Since he had suppression of the right eye, he was hopeful that he did not have to have a further operation.

In conclusion, patients undergoing medial rectus muscle recession in Graves' orbitopathy need to be informed of this adverse complication.

\section{References}

[1] W. A. Lagrèze, J. Gerling and F. Staubach, "Changes of the Lid Fissure after Surgery on Horizontal Extraocular Muscles," American Journal of Ophthalmology, Vol. 140, No. 6, 2005, pp. 1145-1146.

[2] M. P. Mourits, L. Koornneef, W. M. Wiersinga, M. F. Prummel, A. Berghout and R. van der Gaag, "Clinical Criteria for the Assessment of Disease Activity in Graves' Ophthalmopathy: A Novel Approach,” British Journal of Ophthalmology, Vol. 73, No. 18, 1989, pp.
639-644. doi:10.1136/bjo.73.8.639

[3] H. Kakizaki, N. Umezawa, Y. Takahashi and D. Selva, "Binocular Single Vision Field,” Ophthalmology, Vol. 116, No. 2, 2009, p. 364.

[4] H. Kakizaki, M. Zako, T. Nakano, et al., "Direct Insertion of the Medial Rectus Capsulopalpebral Fascia to the Tarsus," Ophthalmic Plastic \& Reconstructive Surgery, Vol. 24, No. 2, 2008, pp. 126-130. doi:10.1097/IOP.0b013e3181647cb2

[5] H. Kakizaki, D. Selva and I. Leibovitch, "Dynamic Study of the Medial and Lateral Recti Capsulopalpebral Fasciae Using Cine Mode Magnetic Resonance Imaging,” Ophthalmology, Vol. 117, No. 2, 2010, pp. 388-391.

[6] C. D. McCord, C. B. Boswell and T. R. Hester, "Lateral Canthal Anchoring," Plastic and Reconstructive Surgery, Vol. 112, No. 1, 2003, pp. 222-236. doi:10.1097/01.PRS.0000066340.85485.DF

[7] A. K. Eckstein, K. T. Johnson, M. Thanos, J. Esser and M. Ludgate, "Current Insights into the Pathogenesis of Graves' Orbitopathy,” Hormone and Metabolic Research, Vol. 41, No. 6, 2009, pp. 456-464. doi:10.1055/s-0029-1220935 